\title{
Longitudinal space charge debunching and compensation in high-frequency accelerators
}

\author{
L.C. Steinhauer and W. D. Kimura \\ STI Optronics, Inc., 2755 Northrup Way, Bellevue, Washington 98004-1495
}

(Received 2 March 1999; published 12 August 1999)

\begin{abstract}
Space charge debunching is a major issue for future high-gradient, high-frequency accelerator techniques. Space charge will set limits on the maximum six-dimensional phase space density obtainable in optical or plasma based accelerators. These accelerators will have short microbunches a fraction of an optical wavelength, in which space charge debunching is unmitigated by two-dimensional effects. The element of an accelerator system most vulnerable to space charge is the drift space between the prebuncher and the acceleration sections. A self-consistent model coupling the energy and phase modulation in the drift space is developed. It is shown that both space charge effects and coherent energy spread can be offset by adjusting the prebuncher and beam optics parameters. In the accelerator sections, the large relativistically corrected inertia together with two-dimensional effects combine to make space charge debunching unimportant. The analytical results compare well with PARMELA code simulations.

PACS numbers: 41.75.Jv, 29.27.Bd, 29.17.+w
\end{abstract}

\section{INTRODUCTION}

Space charge spreading of high energy electron beams is well known. The spreading is both transverse (defocusing) and longitudinal (debunching). The defocusing tendency can be offset by increasing the magnetic focusing field. Space charge debunching [1-3] is a more pernicious tendency since the offsetting force components of the acceleration fields tend to be weak. Fortunately, in radio frequency (rf) linear accelerators (linac) the debunching force is greatly weakened by two-dimensional (2D) effects, namely, that the longitudinal dimension of the bunch $(\mathrm{mm}$ to $\mathrm{cm})$ is much longer than the beam width $(100 \mu \mathrm{m}$ to $1 \mathrm{~mm})$. The critical parameter is the elongation ratio (length-to-diameter ratio) in the beam rest frame. In typical rf linacs the elongation is much greater than unity so that the longitudinal space charge field, which drives debunching, is greatly muted by $2 \mathrm{D}$ effects. A much different situation arises in future high-gradient, highfrequency accelerators based on optical or plasma based accelerators. There the beam is composed of a series of very short microbunches with longitudinal dimension a fraction of an optical wavelength. The elongation can be comparable to unity or less in the early, low beam energy elements of an accelerator system. For low elongation the microbunches begin to resemble pancakes. Space charge debunching forces are maximized in this quasione-dimensional (1D) geometry. Indeed, the space charge debunching field acting on the electrons at the edge of a microbunch can approach $10^{6} \mathrm{~V} / \mathrm{m}$ (e.g., for $0.2 \mathrm{nC}$ and $3 \mathrm{~mm}$ macrobunch charge and length, $100 \mu \mathrm{m}$ beam radius, and $10 \mu \mathrm{m}$ wavelength). This major difficulty must be reckoned with in the design of high-frequency linacs.

Space charge in a beam drives cold plasma oscillations at the plasma frequency, which is determined by the beam density, the relativistic electron inertia, and, of course, the elongation. If offsetting forces are negligible, inertia is the only factor resisting the space charge force. In drift spaces between the injector and the accelerator, and between accelerator sections, there is no offsetting force. In the accelerator sections a component of the accelerating fields can oppose longitudinal space charge spreading, but is ineffective at high energy in highfrequency accelerators. Since inertia is the only factor resisting space charge debunching, the system must be designed to operate in the inertial regime where the elapsed time is a fraction of a plasma oscillation period.

This paper examines the effects of longitudinal space charge spreading in the quasi-1D (low elongation) and the inertial limits. The basic configuration of a high-frequency accelerator, illustrated in Fig. 1, includes a prebuncher for modulating the $e$-beam energy, a drift section to allow longitudinal compression into microbunches, and a series of acceleration sections. Such a configuration is being tested in the Staged Electron Laser Acceleration (STELLA) experiment at the Brookhaven National Laboratory Accelerator Test Facility [4]. Parameters for this experiment will be used in the analysis; however, the results found here are generally applicable.

The outline of the paper is as follows. Section II presents a simple estimate of space charge effects by setting the inertia of the electrons against space charge forces. The buncher is found to be the element of a highfrequency accelerator that is most vulnerable to space charge debunching because the electron inertia is the smallest there. Section III follows with an analysis of space charge debunching in the buncher. This immediately suggests a relatively easy method to counteract the space charge force by overmodulating the $e$-beam in the prebuncher. A fortuitous by-product of this process is that the energy spread introduced by the prebuncher can be reduced, as suggested by previous authors. In Sec. IV, the basic analytical approach is validated using a more 


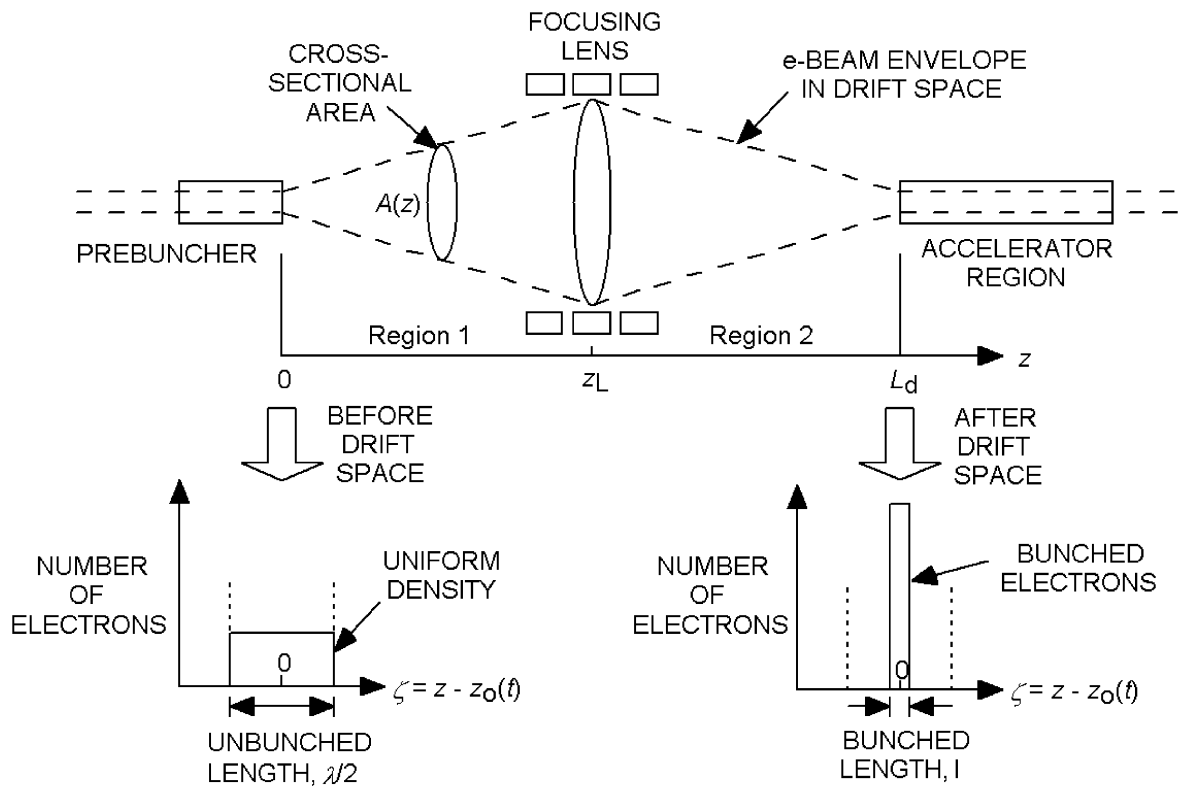

FIG. 1. Idealized microbuncher arrangement.

realistic microbunch distribution and the PARMELA code, which includes space charge effects. Section V concludes with a summary.

\section{ESTIMATE OF SPACE CHARGE EFFECTS}

The simplest estimate of space charge debunching focuses on space charge oscillations. The frequencies of these oscillations in the longitudinal and transverse directions depend on the elongation in the rest frame of the beam

$$
\Re=\gamma l_{\mu} / w,
$$

where $\gamma$ is the relativistic parameter, $l_{\mu}$ is the microbunch length, and $w$ is the beam diameter. (Note that the aspect ratio, as defined elsewhere [5,6], is related to the elongation ratio as $1 / 2 \Re$.) The STELLA experiment offers a practical example from which to extract typical values of the elongation. In the drift space (see Fig. 1) the elongation begins at a relatively large value $(\Re>3)$, but falls rapidly, dropping below unity well before passing the midpoint of the drift distance, and it remains in the range $0.4-0.5$ after the focusing lens. In the acceleration sections that follow, $\Re$ increases as the beam energy increases and exceeds 2 by the end of the acceleration region $(\gamma \sim 300)$. Thus the debunching is roughly 1D over much of the drift space, but 2D effect begins to play a role in the accelerator region.

In the 1D (low elongation) limit, longitudinal space charge forces drive oscillations at the plasma frequency $\omega_{p}=\left(e^{2} n / m_{e} \varepsilon_{0} \gamma^{3}\right)^{1 / 2}$, where $m_{e},-e$, and $n$ are the electron rest mass, charge, and density, respectively, and $\varepsilon_{0}$ is the free-space permittivity. Note that the relativistic correction to the mass for relative longitudinal motion is $\gamma^{3}$. This arises as follows: the rate of change of momentum is $d\left(\beta \gamma m_{e} c\right) / d t$ ( $\beta$ is the relativistic variable, $c$ is the speed of light), since $\beta=\left(1-1 / \gamma^{2}\right)^{1 / 2}$ then $\Delta \gamma=$ $\beta \gamma^{3} \Delta \beta$; thus the momentum change is $m_{e} c \gamma^{3} d \beta / d t$ so that the corrected inertia is $m_{e} \gamma^{3}$. Space charge debunching becomes significant if the transit time $\sim L / \beta c$ ( $L=$ travel distance) is comparable to or exceeds the plasma oscillation time $1 / \omega_{p}$, i.e., if $\omega_{p} L / c \geq O(1)$ for $\beta \sim 1$. Accordingly, we define a space charge parameter

$$
\sigma_{\mathrm{sc}} \equiv \omega_{p}^{2} L^{2} / c^{2}
$$

if $\sigma_{\mathrm{sc}} \geq O(1)$, space charge effects will be significant.

An expression for $\sigma_{\mathrm{sc}}$ is readily found in terms of practical units. The beam properties are the macrobunch length $l_{M}$, charge $Q_{M}$, and the normalized emittance $\varepsilon_{N}$. If $A$ is the cross-sectional area of the beam, then the density is $n=Q_{M} / e A l_{M}$. As a representative area we choose the emittance area

$$
A_{\varepsilon}=\pi \varepsilon_{N} L_{d} / \gamma,
$$

i.e., the geometric mean of the waist area $A_{w}$ and the area at a distance $\sim L$ from the waist (far-field limit $A_{w} \ll A_{\varepsilon}$ ). Combining these with Eq. (2) gives

$$
\sigma_{\mathrm{sc}}=\frac{4 r_{e} L}{\gamma^{2} l_{M} \varepsilon_{N}} \frac{Q_{M}}{e}=7.04 \times 10^{-5} \frac{L(\mathrm{~m}) Q_{M}(\mathrm{nC})}{\gamma^{2} l_{M}(\mathrm{~m}) \varepsilon_{N}(\mathrm{~m} \mathrm{rad})},
$$

where $r_{e}=e^{2} / 4 \pi \varepsilon_{0} m_{e} c^{2}$ is the classical electron radius. This expression can be applied in the buncher, in which case $L \sim L_{d}$, the drift space length. It can also be applied in the acceleration region. Then the appropriate length is the 
mean energy (average of initial and final), divided by the acceleration gradient $L=\bar{W} / W^{\prime}$. Thus, using Eq. (4) and $\gamma=1.957 \mathrm{~W}(\mathrm{MeV})$, the space charge parameter in the acceleration region is

$$
\sigma_{\mathrm{sc}}=1.84 \times 10^{-5} \frac{Q_{M}(\mathrm{nC})}{W^{\prime}(\mathrm{MeV} / \mathrm{m}) \bar{W}(\mathrm{MeV}) l_{M}(\mathrm{~m}) \varepsilon_{N}(\mathrm{~m} \mathrm{rad})} .
$$

The space parameter is examined for conditions relevant to the STELLA experiments [4] and to a future high-gradient, high-frequency accelerator. These are summarized in Table I. In the STELLA buncher, $\sigma_{\mathrm{sc}}=1.2$, indicating that space charge effects are significant and clearly warrant further investigation. In the STELLA accelerator region $\sigma_{\mathrm{sc}}=0.13$, indicating that space charge effects are much less important. This is the result of higher energy and enhanced inertia. In the high-gradient, high-frequency accelerator region $\sigma_{\mathrm{sc}}=0.0015$, inertia is so great that space charge effects are insignificant. Evidently, the most vulnerable element of a high-frequency accelerator is the buncher. This is therefore the object of an analytical model in Sec. III.

\section{ANALYTICAL MODEL OF SPACE CHARGE EFFECTS}

\section{A. Buncher arrangement}

A simple method of compressing into microbunches, shown in Fig. 1, employs a prebuncher [e.g., an inverse free-electron laser (IFEL)] followed by a drift space. The beam enters the prebuncher as a macrobunch with a length many times the laser wavelength $\lambda$. It has very little energy spread initially. The prebuncher gives it an energy modulation with longitudinal periodicity $\sim \lambda$. Within each "segment" of width $\lambda$, electrons behind the centroid of the segment have slightly higher energy and speed, and those ahead have slightly lower. In traversing the drift space (length $L_{d}$ ) the electrons behind the centroid catch up, while those ahead of it fall back. This bunch compression occurs in each segment, producing a series of microbunches, having the appearance of a stack of thin pancakes spaced apart by $\lambda$. While this arrangement produces microbunching, it also introduces a coherent energy spread.

\section{B. One-dimensional microbunching model}

A self-consistent model of the electron energy and phase in a microbunch is developed based on three approximations. (i) Since the elongation of the microbunches in the drift space is comparable to or less than unity in the drift space the geometry is quasi-1D. A simple 2D correction factor, which is presented in Appendix A, can be applied to this. (ii) Additionally, since debunching is the issue being addressed, we need consider only a single microbunch (as in Fig. 1). Space charge fields from other microbunches push the electrons in a given microbunch forward or backward as a unit, but do not cause debunching. Justification for this approximation based on a comparison of analyses for a single microbunch and multiple microbunches is presented in Appendix A. (iii) Finally, a sawtooth modulation of the energy modulation is also assumed, as will be discussed shortly.

Define a phase variable in the moving frame of the microbunch, $\varphi \equiv(2 \pi / \lambda)\left[z-z_{0}(t)\right]$, where $z$ is the longitudinal coordinate, $z_{0}$ is the position of the microbunch centroid, and $t$ is the time. Adopt the alternative "time" variable $z=\bar{\beta} c t$ where $\bar{\beta} c$ is the mean speed of the electrons. Then the evolution of the beam is governed by the equations of continuity, Gauss, and motion,

$$
\begin{gathered}
\bar{\beta} c \frac{\partial}{\partial z}(n A)+\frac{2 \pi}{\lambda} \frac{\partial}{\partial \varphi}\left(n u_{z} A\right)=0, \\
\frac{2 \pi}{\lambda} \frac{\partial E_{z}}{\partial \varphi}=-\frac{e}{\varepsilon_{0}} n, \\
\bar{\beta} \bar{\gamma}^{3} m_{e} c\left(\frac{\partial u_{z}}{\partial z}+\frac{2 \pi u_{z}}{\lambda c} \frac{\partial u_{z}}{\partial \varphi}\right)=-e E_{z},
\end{gathered}
$$

TABLE I. Accelerator parameters.

\begin{tabular}{lccc}
\hline \hline \multicolumn{1}{c}{ Parameter } & $\begin{array}{c}\text { STELLA } \\
\text { buncher }\end{array}$ & $\begin{array}{c}\text { STELLA } \\
\text { accelerator }\end{array}$ & $\begin{array}{c}\text { High-gradient, } \\
\text { high-frequency } \\
\text { accelerator }\end{array}$ \\
\hline Initial energy $W_{i}$ & $45 \mathrm{MeV}$ & $45 \mathrm{MeV}$ & $50 \mathrm{MeV}$ \\
Final energy $W_{f}$ & $45 \mathrm{MeV}$ & $145 \mathrm{MeV}$ & $10 \mathrm{GeV}$ \\
Acceleration gradient $W^{\prime}$ & $\ldots$ & $100 \mathrm{MeV} / \mathrm{m}$ & $500 \mathrm{MeV} / \mathrm{m}$ \\
Drift space length $L_{d}$ & $2 \mathrm{~m}$ & $\cdots$ & $\cdots$ \\
Accelerator length $L_{\mathrm{acc}}$ & $\cdots$ & $1 \mathrm{~m}$ & $20 \mathrm{~m}$ \\
Normalized emittance $\varepsilon_{N}$ & $1 \mathrm{~mm} \mathrm{mrad}$ & $1 \mathrm{~mm} \mathrm{mrad}$ & $1 \mathrm{~mm} \mathrm{mrad}$ \\
Macrobunch charge $Q_{M}$ & $0.2 \mathrm{nC}$ & $0.2 \mathrm{nC}$ & $0.2 \mathrm{nC}$ \\
Macrobunch length $l_{M}$ & $3 \mathrm{~mm}$ & $3 \mathrm{~mm}$ & $1 \mathrm{~mm}$ \\
Space charge parameter $\sigma_{\mathrm{sc}}$ & 1.2 & 0.13 & 0.0015 \\
\hline \hline
\end{tabular}


respectively. The unknowns are the density $n$, the velocity relative to the bunch centroid $u_{z}$, and the electric field $E_{z}$, each of which is a function of $\varphi$ and $z$. The crosssectional area of the beam $A(z)$ (see Fig. 1), depends on the focusing optics and will be described shortly. Consider the self-similar forms

$$
\begin{aligned}
n(z) & =\frac{Q_{M} \lambda}{2 e l_{M}}[A(z) l(z)]^{-1}, \\
u_{z}(z, \varphi) & =\frac{c \lambda}{2 \pi} \frac{1}{l} \frac{d l}{d z} \varphi, \\
E_{z}(z, \varphi) & =-\frac{e \lambda}{2 \pi \varepsilon_{0}} n(z) \varphi .
\end{aligned}
$$

This is the case of linear microbunching $\left(u_{z} \propto \varphi\right)$ corresponding to an ideal sawtooth modulation. Note that the density profile in this self-similar case is "square"; i.e., the density is uniform between the leading and trailing edges of the bunch. These self-similar forms identically satisfy Eqs. (6) and (7), and the equation of motion simplifies to

$$
\frac{d^{2} l}{d z^{2}}=\sigma_{\mathrm{sc}} \frac{\lambda A_{\varepsilon}}{2 L_{d}^{2}}[A(z)]^{-1} .
$$

The initial conditions are as follows. The energy modulation is $\Delta \gamma(\varphi, z)=\gamma-\bar{\gamma}=\bar{\beta} \bar{\gamma}^{3} \Delta \beta=\bar{\beta} \bar{\gamma}^{3} u_{z} / c$. From Eq. (9b), the energy modulation $\Delta \gamma \propto \varphi$ also has a sawtooth form. A more realistic form is sinusoidal, $\Delta \gamma \propto \sin \varphi$. Although the sawtooth form is artificial, it is roughly consistent with the sinusoidal form over half of the phase range, $|\varphi| \leq \pi / 2$. Electrons in the other half of the phase range, $\pi / 2 \leq|\varphi| \leq \pi$, are not bunched. This division of phases between electrons that are bunched and those that are not is characteristic of any periodic modulation, including the sinusoidal form. The unbunched electrons do not contribute to the space charge field in the microbunch. Therefore, the analytic model applies only to the electrons in the bunching range $|\varphi| \leq \pi / 2$. Note that a realistic sinusoidal modulation will be assumed in the PARMELA simulations of Sec. IV; this will offer an a posteriori check on whether the sawtooth approximation of the analytic model is a valid one.

The initial condition on the microbunch width is therefore half the periodicity length,

$$
l(0)=\lambda / 2 .
$$

The initial condition on $d l / d z$ is found as follows. The modulation at the microbunch edge $(\zeta=l / 2)$ is $\Delta \gamma_{\text {edge }}=(1 / c) \beta \gamma^{3}\left(u_{z}\right)_{\text {edge }}=(1 / 2) \beta \gamma^{3} d l / d z$. The root mean square (rms) modulation is $1 / \sqrt{2}$ of the edge value. Therefore the initial condition on the derivative is

$$
\left.\frac{d l}{d z}\right|_{z=0}=-\frac{2 \sqrt{2}}{\bar{\beta} \bar{\gamma}^{3}}\left(\Delta \gamma_{\mathrm{rms}}\right)_{\mathrm{pre}},
$$

where $\Delta \gamma_{\mathrm{rms}}$ is the initial modulation. The minus sign is because the modulation leads to a shortening of the microbunch length. Since half the electrons are microbunched, a factor of $1 / 2$ must be included on the right-hand side of Eq. (10). Then, including the 2D reduction factor (Appendix A),

$$
\frac{d^{2} l}{d z^{2}}=\sigma_{\mathrm{sc}} F_{2 \mathrm{D}} \frac{\lambda A_{\varepsilon}}{4 L_{d}^{2}}[A(z)]^{-1} .
$$

Here we are considering only the case where $l$ does not change sign, i.e., microbunching electrons do not pass the centroid. It is conventional to measure microbunching in terms of the phase spread,

$$
\Delta \phi(z)=2 \pi l(z) / \lambda .
$$

An expression for $A(z)$ is needed to complete the analytic solution. The drift space in Fig. 1 has an idealized focusing lens located at $z=z_{L}$, i.e., the thin lens approximation. In practice the lens would be a triplet of quadrupole magnets occupying a finite length. In the regions before Region 1 and after Region 2, the $e$-beam width $w(z)$, is

$$
w^{2}=W_{i}^{2}+4 \theta_{i}^{2}\left(z-Z_{i}\right)^{2},
$$

where $W_{i}, Z_{i}$ are the waist width and location, and $\theta_{i}$ is the asymptotic divergence angle; the subscripts $(i=1,2)$ denote the two regions. The normalized emittance $\varepsilon_{N}=$ $\gamma W_{i} \theta_{i} / 2$ is the same in both regions. For a circular beam the area function is

$$
A(z)=A_{w i}+\frac{A_{\varepsilon}^{2}}{A_{w i} L_{d}^{2}}\left(z-Z_{i}\right)^{2},
$$

where $A_{w i} \equiv \pi W_{i}^{2} / 4$, and $A_{\varepsilon}$ is the emittance area [Eq. (3)]. The two area functions $(i=1,2)$ must match at the lens $\left(z=z_{L}\right)$. The parameters in Region 1 are set by the prebuncher properties (length $L_{\text {pre }}$ ): the waist is at the center of the prebuncher, $Z_{1}=-L_{\text {pre }} / 2$, and the area at the end of the modulator is twice the waist area, $A(0)=2 A_{w 1}$. The latter is the design choice in the STELLA experiments. Then the maximum area (at the triplet) is

$$
A_{L}=A_{\varepsilon} \frac{L_{\text {pre }}}{2 L_{d}}\left[1+\left(1+\frac{2 z_{L}}{L_{\text {pre }}}\right)^{2}\right] .
$$

The parameters in Region 2 are set by the matching requirement $A\left(z_{L}\right)=A_{L}$ and by the focusing strength of the lens. In place of the latter, we simply specify the second waist location $Z_{2}$. Then the area at the second waist is

$$
A_{w 2}=\frac{A_{L}}{2}\left[1 \pm \sqrt{1-\left(\frac{2 A_{\varepsilon}}{A_{L}} \frac{Z_{2}-z_{L}}{L_{d}}\right)^{2}}\right] .
$$

In most cases the lower sign (-) is appropriate; only for extremely weak focusing (small divergence angle) is the upper sign needed. Figure 2 shows the $e$-beam diameter as a function of distance for typical parameters. In this 


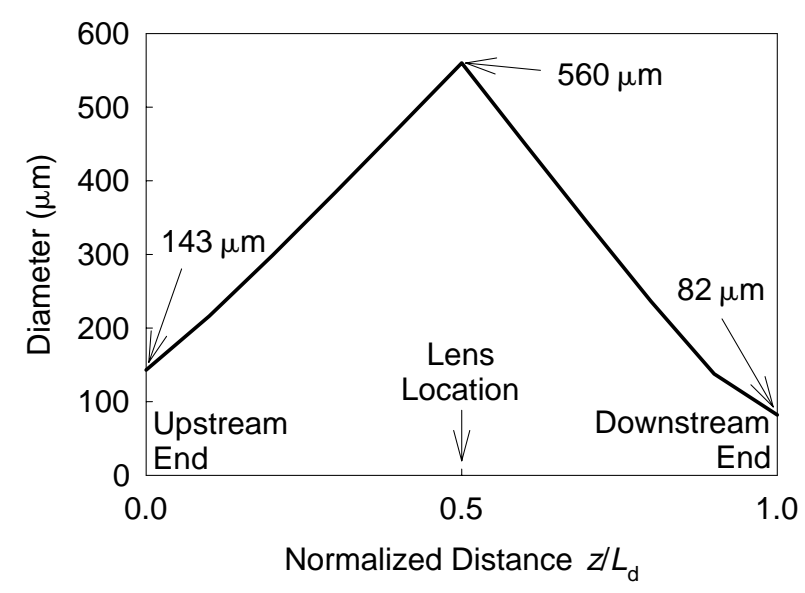

FIG. 2. Beam optics example in the drift space: $\varepsilon_{N}=$ $1 \mathrm{~mm}$ mrad, $\gamma=88, \quad L_{d}=2 \mathrm{~m}, \quad z_{w 1}=-0.235 \mathrm{~m}, \quad z_{T}=$ $1.0 \mathrm{~m}$, and $z_{w 2}=2.0 \mathrm{~m}$.

case the first beam waist is in the modulator section (not shown).

\section{Analytic solution}

The phase and rms energy spreads at the end of the drift space (subscript $f$ ) are

$$
\begin{gathered}
\frac{\Delta \phi_{f}}{\pi}=1-\frac{4 \sqrt{2} L_{d}}{\lambda \bar{\gamma}^{3}} \Delta \gamma_{\mathrm{pre}}+\frac{\sigma_{\mathrm{sc}} F_{2 \mathrm{D}}}{2} K, \\
\frac{\Delta \gamma_{f}}{\bar{\gamma}}=\left|-\frac{\Delta \gamma_{\mathrm{pre}}}{\bar{\gamma}}+\frac{\lambda \bar{\gamma}^{2}}{4 \sqrt{2} L_{d}} \frac{\sigma_{\mathrm{sc}} F_{2 \mathrm{D}}}{2} J\right|,
\end{gathered}
$$

where the relativistic limit $\beta \rightarrow \infty$ is assumed. The right-hand side of these equations represents the space charge effect. The appearance of $\sigma_{\mathrm{sc}}$ in both expressions reflects the self-consistent coupling of the phase $\Delta \phi$ and modulation $\Delta \gamma$. Geometric effects of the beam optics are contained in the $J$ and $K$ factors, which are derived in Appendix B. Note that space charge tends to increase the phase spread [Eq. (14)] and reduce the energy spread introduced by prebunching [Eq. (15)].

Figure 3 shows the dependence of the beam optics factors on the location of the second waist. The nominal values for the factors are $J \sim 2, K \sim 2 / 3$. Their dependence on the optics arrangement (Fig. 3) can be explained as follows. Space charge effects are enhanced in the neighborhood of a beam waist because of the high density there. Thus if the second waist is located before the end of the drift space, then $J$ and $K$ are larger. If the second waist is after the end of the drift space, then no second waist appears in the drift space and $J$ and $K$ are lower. Note that a solid line in Fig. 3 is used when the final beam diameter $\left(z=L_{d}\right)$ is smaller than its initial $(z=0)$ value, and a dashed line when the final diameter is larger. This affects the issue of beam acceptance in the accelerator sections.

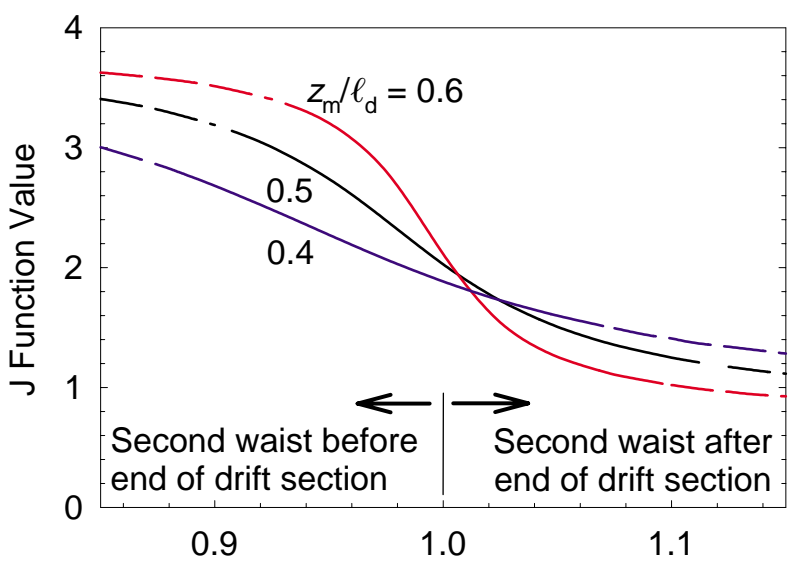

Normalized 2nd Waist Location $Z_{2} / \ell_{\mathrm{d}}$

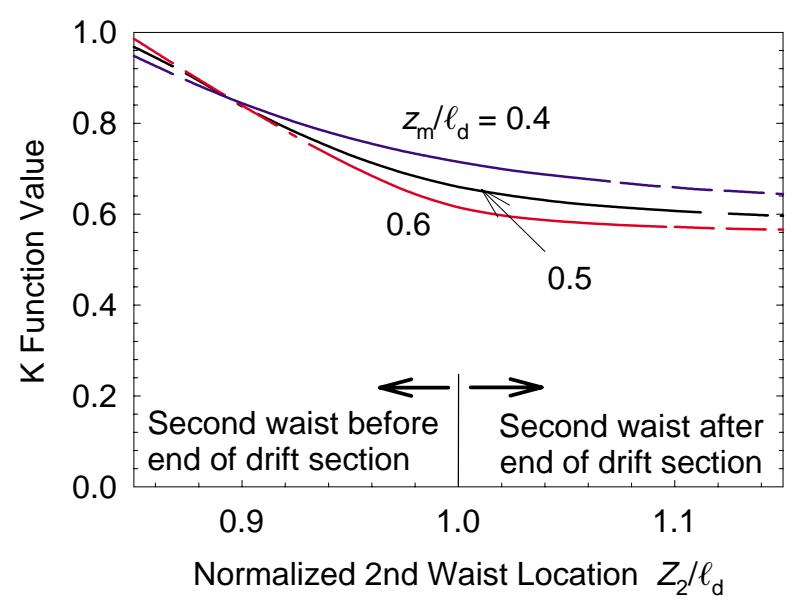

FIG. 3. Geometric factors in coupling between beam optics and space charge. $J$ is the energy spread, and $K$ is the phase spread. All examples have $\varepsilon_{N}=1 \mathrm{~mm} \mathrm{mrad,} \gamma=88$, $L_{d}=2 \mathrm{~m}$, and $z_{w 1}=-0.235 \mathrm{~m}$.

The purpose of the buncher is to create short microbunches, i.e., to make $\Delta \phi_{f}$ as small as possible. In the idealized sawtooth model, this corresponds to $\Delta \phi_{f} \rightarrow 0$. Then from Eq. (14), the prebuncher modulation would be set to

$$
\frac{\Delta \gamma_{\mathrm{pre}}}{\bar{\gamma}}=\frac{\lambda \bar{\gamma}^{2}}{4 \sqrt{2} L_{d}}\left(1+\frac{\sigma_{\mathrm{sc}} F_{2 \mathrm{D}}}{2} K\right) .
$$

The corresponding final energy spread is then

$$
\frac{\Delta \gamma_{f}}{\bar{\gamma}}=\frac{\lambda \bar{\gamma}^{2}}{4 \sqrt{2} L_{d}}\left|1-\frac{\sigma_{\mathrm{sc}} F_{2 \mathrm{D}}}{2}(J-K)\right| .
$$

Since $J>K$, the effect of space charge is to reduce the final energy spread. Indeed, it may be possible in some cases to eliminate the coherent energy spread introduced by the modulator, which occurs if $\sigma_{\mathrm{sc}}=2 /(J-$ $K) F_{2 \mathrm{D}}$. This possibility has been suggested elsewhere; see, e.g., Fig. 5 of Ref. [7]. Therefore, space charge in 
TABLE II. Beam optics in the buncher of STELLA.

\begin{tabular}{lc}
\hline \hline Laser wavelength $\lambda$ & $10.6 \mu \mathrm{m}$ \\
Drift space length $L_{d}$ & $2 \mathrm{~m}$ \\
First waist location $Z_{1} / L_{d}$ & -0.125 \\
Lens location $z_{L} / L_{d}$ & 0.5 \\
Second waist location $Z_{2} / L_{d}$ & 1 \\
\hline \hline
\end{tabular}

combination with an appropriate beam optics design can be exploited to minimize both the microbunch length and energy spread.

Consider a practical example. Table II shows beam optics parameters relevant to the prebuncher in the STELLA experiment [4]. Here the beam widths are 151, 544, and $85 \mu \mathrm{m}$ at the beginning, lens position, and end of the drift space, respectively. In this example the 2D correction factor (see Appendix A) is in the range of $0.75-0.85$ in the region after the focusing lens and, thus, $F_{2 \mathrm{D}}=0.8$ has been chosen as a representative value. That $F_{2 \mathrm{D}} \approx$ constant reflects the fact that the elongation of the microbunches remains about the same in this region. Near the lens the pancakes have a large diameter, but are fairly thick; while near the end, both the diameter and thickness are much reduced, but remain in about the same proportion.

Table III shows examples of the energy spread for optimal microbunching [Eq. (17)]. In the first example (no space charge) the $0.73 \%$ energy spread introduced by the prebuncher remains the same throughout the drift space. In the $0.2 \mathrm{nC}$ example, the prebuncher energy spread must be raised to $0.95 \%$ (a factor of 1.3 higher) to compensate for space charge. In this case the final energy spread is reduced by a factor of 3 by space charge effects. It may be possible to reduce the energy spread further by adjusting the optics arrangements, e.g., by adjusting the triplet and second focus locations.

\section{COMPARISON WITH PARMELA SPACE CHARGE SIMULATION CODE}

The purpose of this section is to validate the analytic approximation by comparison with a series of computations with the PARMELA code [8]. The initial conditions for the PARMELA simulations (phase space distribution of the electrons) are generated by an FEL modeling code [9]

TABLE III. Energy spread in the buncher.

\begin{tabular}{|c|c|c|c|}
\hline $\begin{array}{l}\text { Microbunch } \\
\text { charge } Q_{b}\end{array}$ & $\begin{array}{c}\text { Space charge } \\
\text { parameter } \\
\sigma_{\mathrm{sc}} \\
\end{array}$ & $\begin{array}{c}\text { Prebuncher } \\
\text { energy } \\
\text { spread } \\
\Delta \gamma_{\text {pre }} / \gamma \\
\end{array}$ & $\begin{array}{c}\text { Final energy } \\
\text { spread } \\
\Delta \gamma_{f} / \gamma\end{array}$ \\
\hline No space charge & 0 & $0.73 \%$ & $0.73 \%$ \\
\hline $0.1 \mathrm{nC}$ & 0.61 & $0.84 \%$ & $0.49 \%$ \\
\hline $0.2 \mathrm{nC}$ & 1.21 & $0.95 \%$ & $0.25 \%$ \\
\hline
\end{tabular}

with the IFEL operated well below saturation. This produces an approximately sinusoidal energy modulation of the beam. Note that the FEL modeling code does not include space charge effects; this assumption is reasonable since little bunching takes place within the $47 \mathrm{~cm}$ long IFEL assumed in the simulation. For simplicity the case of nearly constant beam area is considered. The parameters for the FEL modeling code and PARMELA are listed in Table IV. For a more accurate comparison, the analytic model assumes the following: (i) The drift length includes the $2 \mathrm{~m}$ drift space plus half the length of the $0.47 \mathrm{~m}$ long prebuncher (IFEL wiggler in STELLA); this roughly accounts for the partial microbunching that takes place within the prebuncher. (ii) The beam radius of $100 \mu \mathrm{m}$ is roughly the average of the PARMELA example ( $89 \mu \mathrm{m}$ initial to $109 \mu \mathrm{m}$ final). (iii) The macrobunch charge is reduced by a factor of $\left(1-e^{-1}\right)=0.632$ to account for the difference between a sharp edge beam (analytic) and the Gaussian radial profile (PARMELA); this is the fraction of electrons in a Gaussian distribution that lie within the root mean square radius of the beam.

The analytic model developed in Sec. III allows variable beam area. Here it is simplified to the case of constant area. As before, the model is not valid after bunch inversion, i.e., the point when trailing electrons catch up and pass the centroid, and leading electrons fall back past the centroid. Then Eq. (13) becomes

$$
\frac{d^{2} l}{d z^{2}}=\bar{\sigma}\left(\frac{\lambda}{2 L_{d}^{2}}\right),
$$

where $\bar{\sigma} \equiv \sigma_{\mathrm{sc}} F_{2 \mathrm{D}} A_{\varepsilon} / 2 A$ is a modified space charge parameter. For the parameters in Table IV, $\sigma_{\mathrm{sc}} A_{\varepsilon}=$ $3.416 \times 10^{-8} \mathrm{~m}^{2}$, and $A=\pi r_{\text {rms }}^{2}=3.14 \times 10^{-8} \mathrm{~m}^{2}$. For constant area, the $J$ and $K$ functions (Sec. III) simplify to $J=A_{\varepsilon} / A$, and $K=A_{\varepsilon} / 2 A$. By simple integrations, the solution of Eq. (18) for no bunch inversion is

$$
\begin{aligned}
& l_{f}^{\prime}=l_{i}^{\prime}+\bar{\sigma} \lambda / 2 L_{d}, \\
& l_{f}=(1+\bar{\sigma} / 2) \lambda / 2+l_{i}^{\prime} L_{d},
\end{aligned}
$$

TABLE IV. Parameters for the IFEL modeling code and PARMELA.

\begin{tabular}{lc}
\hline \hline \multicolumn{1}{c}{ Parameter } & Value \\
\hline$e$-beam energy & $45 \mathrm{MeV}$ \\
Intrinsic energy spread & $1.5 \times 10^{-4} \%$ \\
Emittance $(x$ and $y)$ & $2 \times 10^{-12} \mathrm{~m} \mathrm{rad}$ \\
Twiss parameter $\alpha_{x}, \alpha_{y}$ & $8000 \mathrm{~m}$ \\
Twiss parameter $\beta_{x}, \beta_{y}$ & 0 \\
Wiggler length & $47 \mathrm{~cm}$ \\
Wiggler wavelength & $3.33 \mathrm{~cm}$ \\
Laser wavelength $\lambda$ & $10.6 \mu \mathrm{m}$ \\
Laser power & $0-8 \mathrm{MW}$ \\
Macrobunch charge & $0.2 \mathrm{nC}$ \\
Microbunch charge & $7 \times 10^{-4} \mathrm{nC}$ \\
Number of electrons & 5000 \\
\hline \hline
\end{tabular}


where the subscripts $i$ and $f$ denote initial and final values, respectively, and the prime denotes the derivative with respect to $z$. This result is valid for $l \geq 0$ (no bunch inversion). It is convenient to express the initial energy spread in terms of the amplitude of the maximum energy spread imparted by the laser; then

$$
\Delta \gamma_{i} / \gamma=-l_{i}^{\prime}\left(\gamma^{2}-1\right) / \pi .
$$

It is also conventional to express the final energy using the root mean square value, which is lower than the amplitude by a factor of $\sqrt{3}$ for a sawtooth modulation,

$$
\Delta \gamma_{f} / \gamma=\left|\tilde{l}_{f}^{\prime}\right|\left(\gamma^{2}-1\right) / \sqrt{3} \pi .
$$

The final phase spread (full width) is $\Delta \phi_{f}=(\pi / 2) l_{f}$.

The foregoing model assumes a constant value of $\bar{\sigma}$. Actually, the 2D correction $F_{2 \mathrm{D}}$ (Appendix A) varies somewhat. At the start of the drift space, the microbunches are fairly thick so that 2D effect may be significant, whereas near the end they are relatively thin (pancakelike), i.e., roughly 1D. By contrast, in the variable area case (Sec. III) the elongation remains low and roughly constant after the focusing lens. Here, as an approximation, we use the average of $F_{2 \mathrm{D}}$ from $\Delta \phi=\pi$ (initial) down to $\Delta \phi_{f}$ (final).

The phase space ( $\Delta W$ vs $\varphi$ ) results for the PARMELA computation and the analytic model are shown in Fig. 4. Important numerical values for these comparisons are shown in Table V. The no-space-charge case, Fig. 4(a), shows the expected differences in the way a sinusoid and a sawtooth "bunch up." In the former the sinusoid is distorted in the usual manner so that the central section envelopes into a vertical stripe; in the latter the "bar" representing the distribution simply rotates. Adding space charge [Fig. 4(b)] causes the output phase distribution to broaden and reduces the energy distribution amplitude.

In the comparisons that follow shortly, the "top 50\%" of the electrons in the PARMELA computation will be selected. These are the half of the electrons with the best bunching (lowest values of $|\varphi|$ ) as marked in Fig. 4 . Take note that one aspect of the PARMELA results can be misleading in the phase space diagram. The distribution of electrons along the vertical "bands" ( $8^{\circ}$ width "bins") is by no means uniform. For example, in the first bin to the right of zero phase position in Fig. 4(b) (bin centered at $\Delta \varphi=+8^{\circ}$ ), most of the electrons are concentrated toward the lower energy end of the band.

Comparisons between the analytic and PARMELA results as a function of initial modulation are shown in Figs. 5 and 6. The analytical predictions are shown as lines and the PARMELA results as symbols. Consider first the energy spread in Fig. 5. For no modulation $\left(\Delta \gamma_{i} / \gamma=0\right)$, the energy spread is caused by space charge alone. For low, but increasing modulation, $\Delta W_{\mathrm{rms}}$ decreases because modulation partially compensates for the space charge effect. At $\Delta \gamma_{i} / \gamma \approx 0.12 \%$, the energy spread is perfectly
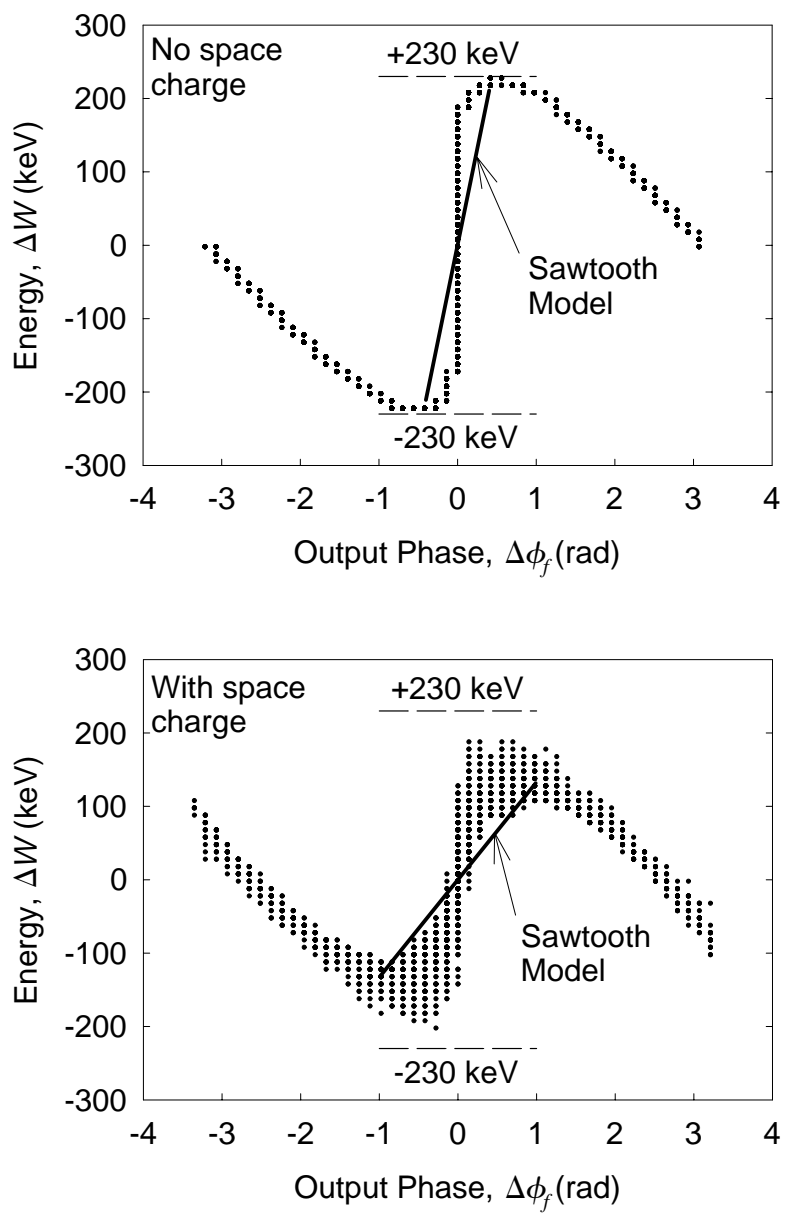

FIG. 4. Phase space diagrams for the analytic and PARMELA results. The initial energy modulation amplitude is $\Delta \gamma / \gamma=$ $0.51 \%$. Other parameters for the PARMELA computation are listed in Table IV. (a) No space charge effects, (b) with space charge effects.

compensated. However, very little bunching (Fig. 6) has occurred at that point $\left(\Delta \phi_{f} \approx 2.8 \mathrm{rad}\right)$. For higher modulation, $\Delta \phi_{f}$ falls monotonically, but $\Delta W_{\text {rms }}$ begins to increase. The latter indicates overcompensation with respect to energy spread. The analytic solution is valid only up to $\Delta \gamma_{i} / \gamma \approx 0.76 \%$ at which point bunch inversion takes place. Evidently the analytic model reproduces the PARMELA results quite well in the range where it applies. The good agreement between the analytical model

TABLE V. Comparison of analytic model and PARMELA computation.

\begin{tabular}{lcc}
\hline \hline & \multicolumn{1}{c}{$\Delta \phi_{f}(\mathrm{rad})$} & $\Delta W_{\mathrm{rms}}(\mathrm{keV})$ \\
\hline No space charge & & \\
$\quad$ Analytic & $0.40\left(\left|\varphi_{i}\right| \leq \pi / 2\right)$ & 138 \\
PARMELA & $0.82($ best $50 \%)$ & 149 \\
With space charge & & \\
$\quad$ Analytic & $0.97\left(\left|\varphi_{i}\right| \leq \pi / 2\right)$ & 76 \\
PARMELA & $1.13($ best $50 \%)$ & 86 \\
\hline \hline
\end{tabular}




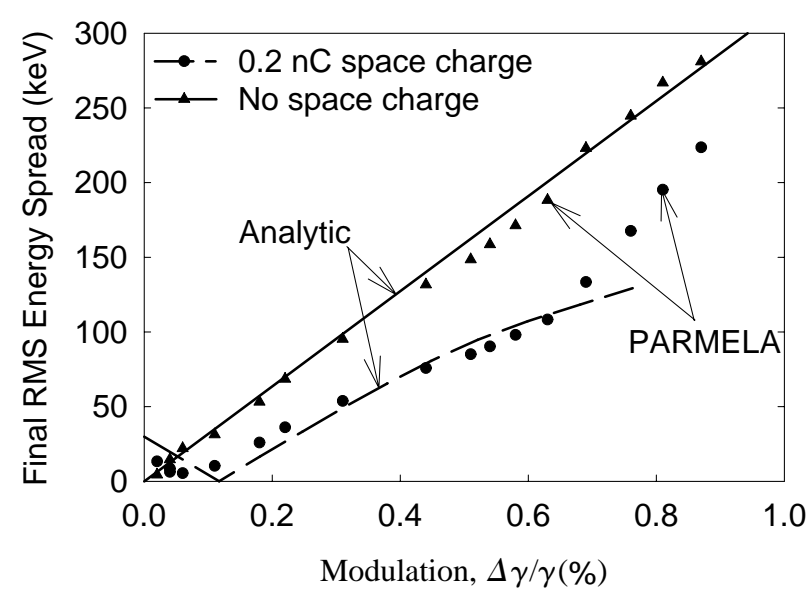

FIG. 5. Comparison between the analytic model and PARMELA code simulations of the final rms energy spread.

(sawtooth modulation) and the PARMELA simulations (sinusoidal modulation) is a posteriori verification that the sawtooth approximation was not critical.

\section{SUMMARY}

Space-charge-induced spreading of an electron microbunch is a potentially important issue in highfrequency accelerators where the microbunches can have longitudinal distributions a fraction of an optical wavelength. The competition between space charge forces and inertial effects can be estimated in terms of the space charge parameter $\sigma_{\mathrm{sc}}$ [Eqs. (2)-(4)]. A significant space charge debunching tendency is likely in a typical buncher ( $\sigma_{\mathrm{sc}}$ of order unity). However, in the acceleration section $\sigma_{\mathrm{sc}}$ is generally quite small so that space charge debunching is insignificant. This is the result of the relativistic increase in electron inertia at high energy.

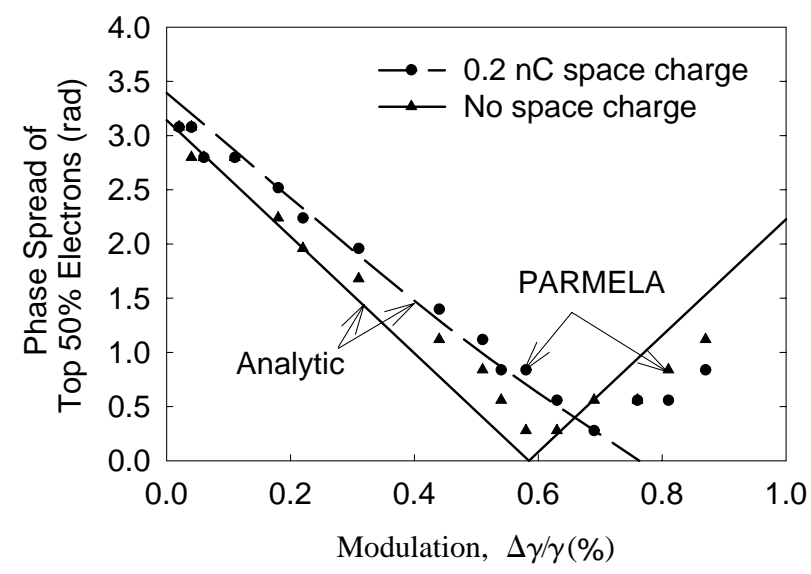

FIG. 6. Comparison between the analytic model and PARMELA code simulations of the phase spread of the top $50 \%$ of the electrons in the center of the microbunch. The analytic model is not valid after bunch inversion [see Eq. (19b)] and, therefore, the dashed line stops at this point.
A quantitative prediction of space charge debunching is found using a quasi-1D model. The 1D approximation is approximately valid in a high-frequency accelerator where the microbunches are like thin pancakes. Realistic beam optics (varying cross-sectional area) is taken into account in the quasi-1D model. Other $2 \mathrm{D}$ effects are added using a correction factor.

The analytic model highlights the basic tendencies of space charge effects. (i) Space charge debunching can be compensated for by overmodulation in the prebuncher, i.e., by increasing the energy modulation introduced there. A few tenths of a percent overmodulation is sufficient in typical systems. (ii) Space charge forces naturally reduce the coherent energy spread introduced in the prebuncher. (iii) By proper design of the beam optics (lens location, focusing strength) it may be possible to simultaneously achieve strong bunching and eliminate the energy spread introduced in the prebuncher. (iv) Using a reference case, the analytic model was compared with computations using the PARMELA code. The results showed that the analytic model reproduces the gross features and many of the details found by the computation. This exercise accomplished the dual purposes of verifying that the analytic model is detailed enough to account for the basic phenomena, and assuring that PARMELA computations are suitable for high-frequency applications with very short bunching lengths.

In brief then, space charge effects can readily be compensated for in high-frequency accelerators by simple measures. Future work will apply PARMELA computations in parallel with the analytic model to predict the detailed space charge effects in the STELLA experiments.

\section{ACKNOWLEDGMENTS}

The authors would like to acknowledge helpful conversations with J.R. Fontana, R.H. Pantell, and J.C. Gallardo. We would also like to thank C. G. Parazzoli for his valuable advice on using the PARMELA code. This work is supported by the U.S. Department of Energy, Grant No. DE-FG03-98ER41061.

\section{APPENDIX A: TWO-DIMENSIONAL CORRECTION}

The 2D correction on the debunching electric field has been modeled by others. Barletta et al. [7] assumed a charge density with a square radial profile and a Gaussian longitudinal distribution. Serafini [10] assumed a cylindrical bunch with a uniform charge density. The results from both are quite close and can be approximated by the expression

$$
F_{2 \mathrm{D}} \approx 1 /(1+0.58 \Re)^{2},
$$

where $\Re$ is the elongation ratio [Eq. (1)]. This expression is accurate to within $4 \%$ for $\Re<3$. 
These analyses neglect the influence of all other microbunches in the series, which are separated from the microbunch in question by integer multiples of the wavelength. Roughly speaking, the electrons in a given microbunch are pushed forward or backward by space charge fields from other microbunches; since they are pushed more-or-less as a unit, there is little debunching effect. This can be verified by a comparison between the single microbunch analysis [Eq. (A1)] and one including the other microbunches. This has been done by assuming an infinite train of microbunches and applying a Fourier analysis [6]. Retaining the leading term in the Fourier expansion gives the 2D correction for a train of microbunches: $\left(F_{2 \mathrm{D}}\right)_{t}=1-x K_{1}(x)$, with $x=k w / 2 \gamma$ and $k=2 \pi / \lambda$. Note that if $l_{\mu} \propto 1 / k$ then $x \propto 1 / \mathfrak{R}$. For a particular phase width of $\Delta \phi=2 \operatorname{rad}\left(l_{\mu}=2 / k\right)$, the microbunch train result $\left(F_{2 \mathrm{D}}\right)_{t}$ is quite close in value to $F_{2 \mathrm{D}}$ [Eq. (A1)], differing by less than $20 \%$ over a broad range of $\Re$. The difficulty with the microbunch train result is that it effectively chooses a particular microbunch phase width $\Delta \phi$. This is a consequence of retaining only the leading term of the Fourier expansion.

\section{APPENDIX B: ANALYTIC SOLUTION IN BUNCHER}

The microbunch length is governed by Eq. (13), subject to the initial conditions Eqs. (11) and (12). The two parts of the solution match at the lens as follows. Adopt as reference quantities $l_{R}=\lambda / 2, A_{R}=A_{\varepsilon}$, and define dimensionless quantities using the tilde $\tilde{z}=z / L_{d}$, $\tilde{z}_{L}=z_{t} / L_{d}, \quad \tilde{z}_{w i}=z_{w i} / L_{d}, \tilde{A}(0)=A(0) / A_{\varepsilon}, \tilde{A}\left(L_{d}\right)=$ $A\left(L_{d}\right) / A_{\varepsilon}, \tilde{A}_{L}=A_{L} / A_{\varepsilon}$, and $\tilde{A}_{w i}=A_{w i} / A_{\varepsilon}$. Then the differential equation and initial conditions become

$$
\begin{gathered}
\frac{d^{2} \tilde{l}}{d \tilde{z}^{2}}=\frac{\sigma_{\mathrm{sc}}}{2 \tilde{A}_{w i}}\left[1+\left(\frac{\tilde{z}-\tilde{z}_{w i}}{\tilde{A}_{w i}}\right)^{2}\right]^{-1}, \\
\tilde{l}(0)=1, \\
\left(\frac{d \tilde{l}}{d \tilde{z}}\right)_{\tilde{z}=0}=\frac{2 L_{d}}{\lambda} \frac{\Delta \gamma_{\mathrm{mod}}}{\bar{\beta} \bar{\gamma}^{3}} .
\end{gathered}
$$

The solution simplifies if general expressions are used covering each region. In each region the "initial" conditions at $\tilde{z}_{i}$ are $\tilde{l}\left(\tilde{z}_{i}\right)=\tilde{l}_{i},(d \tilde{l} / d \tilde{z})_{\tilde{z}_{i}}=\tilde{l}_{i}^{\prime}$. Of course, the actual initial conditions apply to Region 1 where $\tilde{z}_{1}=0$, $\tilde{l}_{1}=1$, and $\tilde{l}_{1}^{\prime}=2 L_{d} \Delta \gamma_{\mathrm{pre}} / \lambda \bar{\beta} \bar{\gamma}^{3}$. In Region 2 the initial conditions are at the end of the drift section, $\tilde{z}_{2}=L_{d}$. These are not known a priori, but will be found later by requiring agreement between $\tilde{l}$ and $d \tilde{l} / d \tilde{z}$ for the two regional solutions where they join at $\tilde{z}_{L}$.

The first integral of Eq. (B1), with the initial condition in Region $i$, is

$$
\frac{d \tilde{l}}{d \tilde{z}}=\tilde{l}_{i}^{\prime}+\frac{\sigma_{\mathrm{sc}}}{2}\left[\tan ^{-1}\left(\frac{\tilde{z}-\tilde{z}_{w i}}{\tilde{A}_{w i}}\right)-\tan ^{-1}\left(\frac{\tilde{z}_{i}-\tilde{z}_{w i}}{\tilde{A}_{w i}}\right)\right] .
$$

Then the derivative at the focusing lens location can be expressed in simplified form as

$$
\tilde{l}_{L}^{\prime}=\tilde{l}_{i}^{\prime}+\left(\sigma_{\mathrm{sc}} / 2\right) J_{i},
$$

where

$$
J_{i}=\tan ^{-1}\left(\frac{\tilde{z}_{L}-\tilde{z}_{w i}}{\tilde{A}_{w i}}\right)-\tan ^{-1}\left(\frac{\tilde{z}_{i}-\tilde{z}_{w i}}{\tilde{A}_{w i}}\right) .
$$

The second integral taken over the range $\tilde{z}_{i}$ to $\tilde{z}_{L}$ with the initial condition in Region $i$ is

$$
\tilde{l}_{T}=\tilde{l}_{i}^{\prime}\left(\tilde{z}_{L}-\tilde{z}_{i}\right)+\left(\sigma_{\mathrm{sc}} / 2\right) L_{i}
$$

where

$$
L_{i}=\left(\tilde{z}_{L}-\tilde{z}_{w i}\right) J_{i}-\frac{\tilde{A}_{w i}}{2} \ln \left\{\frac{1+\left[\left(\tilde{z}_{L}-\tilde{z}_{w i}\right) / \tilde{A}_{w i}\right]^{2}}{1+\left[\left(\tilde{z}_{i}-\tilde{z}_{w i}\right) / \tilde{A}_{w i}\right]^{2}}\right\} .
$$

These solutions [Eqs. (B5) and (B7)] are matched at the lens location. The matching of the derivatives implies

$$
\tilde{l}_{2}^{\prime}-\tilde{l}_{1}^{\prime}=\left(\sigma_{\mathrm{sc}} / 2\right) J
$$

where $J=J_{1}-J_{2}$. The matching of the values of $\tilde{l}$ at the lens implies

$$
\tilde{l}_{2}-\tilde{l}_{1}^{\prime}-1=\left(\sigma_{\mathrm{sc}} / 2\right) K
$$

(recognizing that $\tilde{l}_{1}=1$ ) where $K=K_{1}-K_{2}$, with

$$
K_{i}=\left(1-\tilde{z}_{w i}\right) J_{i}-\frac{\tilde{A}_{w i}}{2} \ln \left\{\frac{1+\left[\left(\tilde{z}_{L}-\tilde{z}_{w i}\right) / \tilde{A}_{w i}\right]^{2}}{1+\left[\left(\tilde{z}_{i}-\tilde{z}_{w i}\right) / \tilde{A}_{w i}\right]^{2}}\right\} .
$$

[1] P. Brunet and S. Buffet, in Linear Accelerators, edited by P.M. Lapostolle and A.L. Septier (North-Holland, Amsterdam, 1970), p. 242.

[2] J. Haimson, in Linear Accelerators (Ref. [1]), p. 435.

[3] M. C. Crowley-Milling, in Linear Accelerators (Ref. [1]), p. 509.

[4] W. D. Kimura, M. Babzien, I. Ben-Zvi, L. P. Campbell, D. B. Cline, R. B. Fiorito, J. C. Gallardo, S. C. Gottschalk, P. He, K.P. Kusche, Y. Liu, R. H. Pantell, I. V. Pogorelsky, D. C. Quimby, K.E. Robinson, D. W. Rule, J. Sandweiss, J. Skaritka, A. van Steenbergen, L. C. Steinhauer, and W. Yakimenko, in Advanced Accelerator Concepts: Eighth Workshop, edited by W. Lawson, C. Bellamy, and D. R. Brosius, AIP Conf. Proc. No. 472 (AIP, New York, 1999), p. 563.

[5] L. Serafini and J.B. Rosenzweig, in Proceedings of the 1997 Particle Accelerator Conference, Vancouver, Canada (IEEE, Piscataway, NJ, 1998), p. 2876. 
[6] J. Rosenzweig, C. Pellegrini, L. Serafini, C. Ternieden, and G. Travish, Nucl. Instrum. Methods Phys. Res., Sect. A 393, 376 (1997).

[7] W. A. Barletta, G. Bellomo, R. Bonifacio, R. Corsini, L. De Salvo, P. Pierini, and M. Pullia, Nucl. Instrum. Methods Phys. Res., Sect. A 329, 348 (1993).
[8] L. M. Young, Los Alamos National Laboratory Report No. LA-UR-96-1835, 1998.

[9] D.C. Quimby, J.M. Slater, and J.P. Wilcoxon, IEEE J. Quantum Electron. 21, 979 (1985).

[10] L. Serafini, IEEE Trans. Plasma Sci. 24, 421 (1996). 